\title{
Differential optical feedback interferometry for the measurement of nanometric displacements
}

\section{Interferometría láser retroalimentada diferencial para la medición de desplazamientos nanométricos}

\author{
Francisco J. Azcona ${ }^{(*, S)}$, Reza Atashkhooei, Santiago Royo ${ }^{(\mathrm{S})}$ \\ Centro de Desarrollo de Sensores, Instrumentación y Sistemas, Universitat Politècnica de Catalunya (CD6-UPC), \\ Rambla St. Nebridi 10, 08222, Terrassa, Spain \\ (*) Email: $\underline{\text { francisco.javier.azcona@cd6.upc.edu }}$ \\ S: miembro de SEDOPTICA / SEDOPTICA member \\ Received / Recibido: 20/09/2013. Revised / Revisado: 24/01/2014. Accepted / Aceptado: 13/02/2014. \\ DOI: http://dx.doi.org/10.7149/OPA.47.1.19
}

\begin{abstract}
:
We have recently proposed differential optical feedback interferometry as a convenient method to measure nanometric displacements. In this paper, we present experimental results to support the proposed method. The acquisition system (in particular the conditioning electronics), and, the signal processing algorithm applied to the captured signal, will be described. Obtained results show good agreement with measurements performed using a capacitive sensor employed as reference.
\end{abstract}

Key words: Optical Feedback Interferometry, High Resolution, Nanometric Displacements, Differential Measurements, Nanotechnology.

\section{RESUMEN:}

Recientemente hemos propuesto la interferometría láser realimentada diferencial como un método para la medición de desplazamientos en la escala nanométrica. En este artículo, presentamos algunos resultados experimentales que validan la teoría previamente presentada. Se describen además, en detalle el sistema de adquisición de datos (en particular la etapa de acondicionamiento de señal) y el algoritmo de procesamiento de señal. Los resultados obtenidos muestran buena concordancia con las mediciones realizadas con un sensor capacitivo utilizado como referencia.

Palabras clave: Interferometría Láser Realimentada, Alta Resolución, Desplazamiento Nanométrico, Medición Diferencial, Nanotecnología.

\section{REFERENCES AND LINKS / REFERENCIAS Y ENLACES}

[1]. R. Lang, K. Kobayashi, "External optical feedback effect on semiconductor injection laser properties", IEEE J. Quantum Electron. 16, 347-355 (1980). DOI

[2]. G. A. Acket, D. Lenstra, A. J. D. Boef, B. H. Verbeek, "The influence of feedback intensity on longitudinal mode properties and optical noise in index guided semiconductor lasers", IEEE J. Quantum. Electron. 20, 1163-1169 (1984). DOI

[3]. W. M. Wang, W. J. O. Boyle, K. T. V. Grattan, A. W. Palmer, "Self-mixing interference in a diode laser: Experimental observations and theoretical analysis", Appl. Opt. 32, 1551-1558 (1993). DOI

[4]. S. Donati, L. Falzoni, S. Merlo, "A PC-interfaced, compact laser-diode feedback interferometer for displacement measurements", IEEE Trans. Instrum. Meas. 45, 942-947 (1994). DOI

[5]. M. Norgia, S. Donati, "A displacement measuring instrument utilizing self-mixing interferometry", IEEE Trans. Instrum. Meas. 52, 1765-1770 (2003). DOI

[6]. L. Scalise, Y. Yu, G. Giuliani, G. Plantier, T. Bosch, "Self-mixing laser diode velocimetry: application to vibration and velocity measurement", IEEE Trans. Instrum. Meas. 53, 223-232 (2004). DOI 
[7]. M. Norgia, G. Giuliani, S. Donati, "Absolute distance measurement with improved accuracy using laser diode self-mixing in a closed loop", IEEE Trans. Instrum. Meas. 56, 1894-1899 (2007). DOI

[8]. C. Bes, G. Plantier, T. Bosch, "Displacement measurements using a self-mixing laser diode under moderate feedback", IEEE Trans. Instrum. Meas. 55, 1101-1105 (2006). DOI

[9]. N. Servagent, T. Bosch, M. Lescure, "Design of a phase-shifting optical feedback interferometer using an electro-optic modulator", IEEE J. Sel. Quantum Electron. 6, 798-802 (2000). DOI

[10]. L. Wei, J. Xi, Y. Yu, J. Chicharo, "Phase unwrapping of self-mixing signals observed in optical feedback interferometry for displacement measurement”, Proceedings of ISPACS 2006, pp. 780-783 (2006).

[11].S. Royo, R. Atashkhooei, F. J. Azcona-Guerrero, "A method of measuring a displacement related parameter using a laser self-mixing measuring system and a laser self-mixing measuring system", PCT Patent 2012/049561 (2012).

[12].F. J. Azcona , R. Atashkhooei, S. Royo, J. Méndez Astudillo, A. Jha, "A method to measure sub nanometric amplitude displacements based on optical feedback interferometry", Proc. SPIE 8789, 878908 (2013). DOI

[13]. G. Plantier, C. Bes, T. Bosch, "Behavioral model of a self-mixing laser diode sensor", IEEE J. Quantum Electron. 41, 1157-1167 (2005). DOI

[14].U. Zabit, F. Bony, T. Bosch, A. D. Rakic, "A self-mixing displacement sensor with fringe-loss compensation for harmonic vibrations", IEEE Photon. Technol. Lett. 22, 410-412 (2010). DOI

[15].F. J. Azcona, R. Atashkhooei, S. Royo, J. Méndez Astudillo, A. Jha, "Differential optical feedback interferometry: A new method to measure nanometer displacements", Libro de Comunicaciones, VIII Reunión Española de Optoelectrónica - Optoel2013, pp. 101-105 (2013).

[16]. S. Donati, "Responsivity and noise of self-mixing photo-detection schemes", IEEE J. Quantum Electron. 47, 1428-1433 (2011). DOI

[17]. E. Kreizig, "Métodos numéricos en general", pp. 399-455 in Matemáricas Avanzadas para Ingeniería Vol. II, $3^{\text {rd }}$ Edt., Limusa Wiley, Mexico (2004).

\section{Introduction}

Optical feedback interferometry (OFI), also known as self-mixing interferometry (SMI), is a well known technique which can be applied to the characterization of displacement related phenomena. Early studies on the subject dated from the 1980s, when several research groups [1-3] studied the behavior of laser diodes (LD) subjected to optical feedback. These early studies gave rise to a series of applications being the most representatives, the measurement of micrometric displacements [4,5], velocity [6] and absolute distance [7].

In the case of OFI displacement measurements, different groups have proposed various methods to increase the maximum attainable resolution with the technique. Such efforts [8-10] have shown an increase on the attainable resolution of the technique in a range that can go up to $\lambda / 25$ in the best cases. Nevertheless, these methods are always restricted to displacements larger than $\lambda / 2$, thus, leaving a measuring gap for smaller displacements.
More recently [11,12], we proposed a differential method capable of reaching theoretical resolutions in the order of $\lambda / 1000-\lambda / 4000$. In this paper we present some of the experimental results obtained with the proposed method. In the next section we include a brief summary of the main theoretical aspects of the differential optical feedback interferometry (DOFI) technique. In Section 3, we describe some of the technical issues that may be found out in the application of DOFI with Fabry-Perot (FP) LDs and we propose an electronic circuit that reduces the described effects. Section 4 is a short summary of experimental results obtained with the method and a comparison with theoretical results. As it will be shown, a decrease in the order of 10 has been found on the attainable resolution when theoretical predictions are compared to experimental results. 


\section{Theoretical background}

Optical feedback interferometry may be described as, a modulation of the optical output power (OOP) of a $L D$, caused by the interaction of the laser light backscattered from a vibrating target, with the standing wave within the laser cavity. Depending on the amount of retroinjected energy, the OOP can take different signal shapes as it is described in [13].

In the case of displacement measurements, it is preferred to work within the weak or moderate feedback level regimes. Working within these regimes allows the acquisition of displacement amplitude and direction with a resolution of $\lambda / 2$. Moreover, by working close to the boundary between the weak and moderate regimes (with a feedback factor $C \approx 1$ ), the risk of fringe loss caused by hysteresis [14] is minimized while increasing the SNR of the acquired signal, therefore, reducing the likelihood of transition misdetection when an automated fringe counting algorithm is used.

DOFI is a technique capable of measuring displacements smaller than $\lambda / 2$ by comparing two OFI signals subjected to a known reference motion. The typical DOFI setup is illustrated in Fig. 1, where, a reference laser $L_{r}$ is placed pointing to a stable linear motion device (e.g. a piezoelectric linear stage PZT), and a second laser (measurement laser $L_{m}$ ), which is attached to the motion stage, aims to the measurement target.

In the case of a static target, and considering that both lasers were capable of emitting to the same wavelength (ideal case), it should be noted that, for any reference displacement of amplitude $A>\lambda$ both OFI signals will have the same number of transitions. Moreover, the time intervals $\left(\Delta t_{x}\right)$ between transitions in both

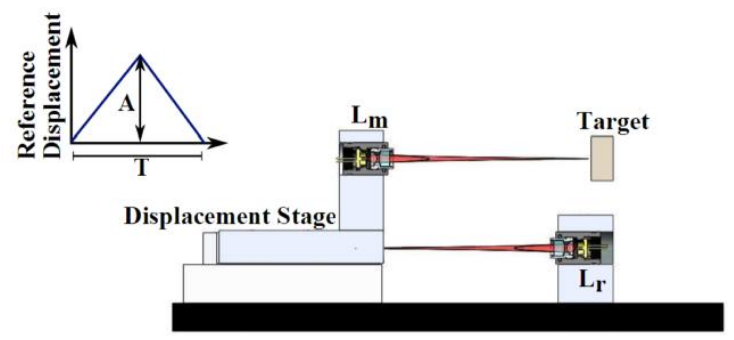

Fig. 1: DOFI setup signals should be equal during the same part of the reference motion. Furthermore, if we consider the case where the target moves with an amplitude $\Delta D \ll \lambda / 2$ (where $\Delta D$ is the target displacement along the direction of the measurement), then, a small time variation between the intervals produced in the reference and measuring signals may be detected, as shown in Fig.2. By using this time difference, it is possible to estimate the amplitude and sense of the target motion with very high resolution.

There are two main factors that need to be considered in the design of the setup before applying the technique: the sampling frequency and the amplitude resolution.

Regarding the sampling frequency, it is important to remark that, the sampling rate of DOFI is different from the acquisition rate used to capture each OFI signal. In DOFI the sampling rate is defined as the time difference between the appearance of two transitions in the reference OFI signal. Therefore, in order to generate a constant sampling rate, the ideal reference motion should be ramp-like. Because of mechanical constraints, as well as to avoid speckle effect over the OFI signal, the ramp-like displacement, described before, is substituted in practice by a periodic triangular motion of limited amplitude (typically lower or equal to 38 $\mu \mathrm{m})$. Taking into account that each transition happens whenever a $\lambda / 2$ displacement is reached, it is possible to define the sampling frequency $\left(f_{s}\right)[12]$ as:

$$
f_{s}=\frac{2 v}{\lambda_{r}}=4 A \frac{f_{r}}{\lambda_{r}}
$$

where $v$ is the stable velocity on a ramp-like displacement, $\lambda_{r}$ the wavelength of $L_{r}$, A the peak to peak amplitude of the reference displacement, and $f_{r}$ the frequency of the reference motion.

Concerning the attainable resolution in amplitude, two factors directly affect the quality of the measurement: the wavelength and the data acquisition rate, which we define as $f_{a}=1 / \Delta t_{\text {min }}$, where $\Delta t_{\text {min }}$ is the sampling time step of the analogue to digital converter (ADC) used for digitizing the signal. For this analysis, we no longer assume that both wavelengths $\left(\lambda_{r}\right.$ 


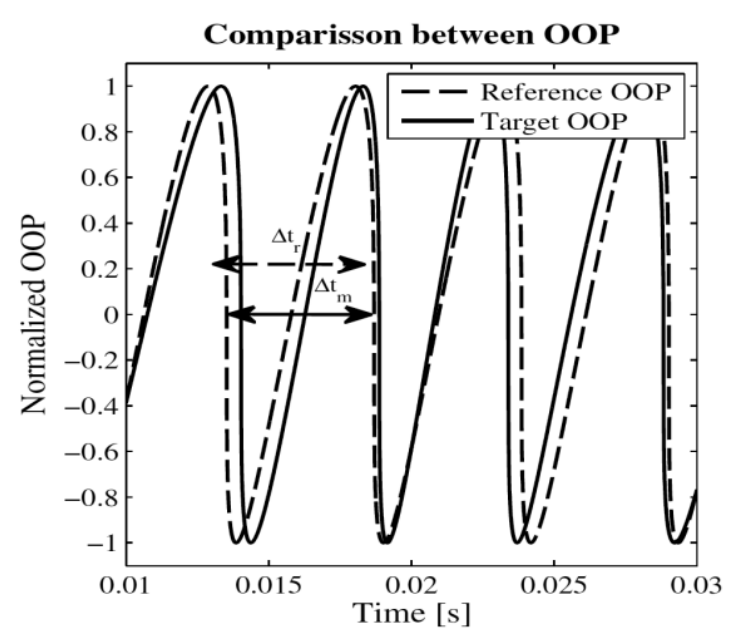

Fig. 2. Time difference between two OFI signals subject to the same reference displacement.

and $\lambda_{m}$ ) are equal. This results in a small time difference in the transition intervals of the signals, which is proportional to the difference of the LD wavelengths. To avoid this bias, it is possible to compare the velocity of the reference stage rather than the direct measurement of DSMI displacement as described in [12].

In order to derive the resolution let us first consider a static target, thus with velocity $\Delta v=0$. For a reference linear displacement (larger than $\lambda$ ), and, by measuring within the time span at which two consecutive transitions happen for each interferometric signal, we can equate:

$$
\Delta v=\frac{\lambda_{m}}{2 \Delta t_{m_{0}}}-\frac{\lambda_{r}}{2 \Delta t_{r}}=0,
$$

from which, if we consider $\lambda_{m}=\lambda_{r}+\Delta \lambda$, it is possible to asses the theoretical time interval between two fringes for a null velocity in the measurement signal $\left(\Delta t_{m_{0}}\right)$ as:

$$
\Delta t_{m_{0}}=\Delta t_{r}\left(1+\frac{\Delta \lambda}{\lambda_{r}}\right) \text {. }
$$

Now, let us consider a small displacement of the target in a quantity lower than $\lambda / 2$. Therefore, a small time differential $\Delta t=$ $\Delta t_{m}-\Delta t_{m_{0}}$ will appear on the measurement signal, leading to:

$$
\Delta v=\frac{\lambda_{m}}{2\left(\Delta t_{m_{0}}+\Delta t\right)}-\frac{\lambda_{r}}{2 \Delta t_{r}} .
$$

By replacing Eq. (3) in Eq. (4) we can arrive to:

$$
\Delta v=-\frac{\lambda_{r}}{2} \frac{\Delta t}{\Delta t_{r}^{2}\left(1+\frac{\Delta \lambda}{\lambda_{r}}\right)+\Delta t \Delta t_{r}}
$$

which, by time integration in the span $\Delta t_{r}$ results in:

$$
\Delta D=-\frac{\lambda_{r}}{2} \frac{\Delta t}{\Delta t_{r}\left(1+\frac{\Delta \lambda}{\lambda_{r}}\right)+\Delta t} .
$$

It should be noted that the equation derived above is sensitive to the direction of the reference motion. For the derivation, we have considered as positive sense the direction in which the reference moves towards the target. In such a case, it can be easily predicted that a displacement of the target in the same direction results in a negative $\Delta t$, therefore, leading to a positive $\Delta D$. In the case of a negative reference motion, a sign inversion of the Eq. (5) and Eq. (6) has to be performed to keep the same sense of direction during the calculation.

For small displacements, where $\Delta t \ll \Delta t_{m_{0}}$ and having a $\Delta \lambda \ll \lambda_{r}$, it is possible to simplify the equation into:

$$
\Delta D \approx-\frac{\lambda_{r}}{2} \frac{\Delta t}{\Delta t_{r}} .
$$

which, for a $\lambda_{r}=785 \mathrm{~nm}, \Delta t_{r} \approx 5.16 \times 10^{-3} s$ and $\Delta t \approx 4 \times 10^{-3} s$ yields a theoretical resolution in the order of $3 \AA$.

Hence, the resolution of the method is limited by the minimum sampling time of the acquisition device $\left(\Delta t_{\text {min }}\right)$, and by the rate of change between transitions on the reference, making it necessary to keep a compromise between sampling frequency $\left(f_{s}\right)$ and resolution.

\section{Signal conditioning and acquisition}

As it can be seen from Eq. (5) and Eq. (6), wavelength plays a crucial role in the DOFI measurement scheme. Any deviation or instability in the wavelength value, during a long time measurement, introduces errors in the estimation of velocity and displacement, hence reducing the resolution and repeatability of the method. Even when the equation introduces a correction factor for the difference in wavelength between both lasers, it does not provide any correction for the variability which 
each wavelength may suffer due to time dependent effects such as temperature and/or current. Thus, it is necessary to use a robust electronic driver able to minimize the transient effects on the wavelength.

Depending on the type of LD used (FP or VCSELs), different approaches are possible. In this work only the FP LD case will be studied, since this type of laser has worse wavelength stability characteristics when compared to VCSELs, due to its higher output power and current threshold.

Two electronic schemes are suitable for the acquisition of the OFI signal as described by Donati [16]. The photodiode (PD) acquisition approach was chosen because of its higher sensitivity when compared to the so-called junction voltage approach. This type of approach, however, does not allow to directly use the PD output as a sensor of the emitted wavelength, which is obtained from the driving current in the laser. Thus, it is necessary either to use an external sensor capable of providing feedback on the wavelength to the laser driver in order to close the loop and stabilize the wavelength of emission, (which would increase the complexity of the method, and also its size and cost); or, reproduce the PD signal to use it as feedback for the laser driver while it is still extracting the PD signal as output for the measurement.

Considering that the measurements to be performed were of relatively low frequency, and taking into account the description of SMI noise sources for both schemes [16], it was decided that reproducing the PD signal was a better option. An electronic card with the basic electronic elements represented on Fig. 3 was assembled. The card is composed by a laser driver which receives feedback from the PD signal after being duplicated at a current mirror. For OFI signal extraction purposes, the PD current is fed into a transimpedance amplifier (TIA). After this stage, the signal is high-pass filtered to recover only the $\mathrm{AC}$ component of the OOP thus obtaining the OFI signal. Finally, the signal is fed into a gain amplifier stage (GA) calibrated for the resolution requirements of the ADC applied on the measurement. Due to the OFI

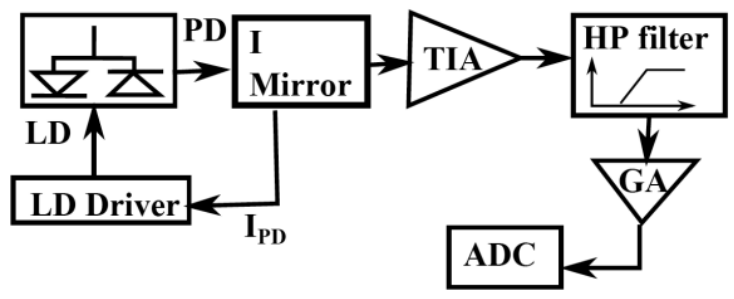

Fig. 3. Schematic representation of electronic card requirements for DOFI acquisition with wavelength stabilization.

signal characteristics, it is necessary to keep a bandwidth as high as possible (typically for low frequency applications from $100 \mathrm{kHz}$ to $1 \mathrm{MHz}$ ).

After signal conditioning, the signal should be recovered by an ADC with high acquisition rate. Depending of the frequency studied, the acquisition rate can be varied from a few $\mathrm{kHz}$ to several $\mathrm{MHz}$ in the case of high frequency reconstruction. A low pass filter may be also applied before acquisition to reduce the noise present in high frequency bands, not used for measurement.

After acquisition, signals are subjected to a Fourier band-pass filtering in order to minimize false detection cases in the fringe counting algorithm. Once the fringes are detected, an algorithm using the velocity and displacement calculations described in Section 2 is applied followed by numerical integration using trapezoidal rule [17]. Finally, linear interpolation using Newton forward difference formula [17] between two consecutive measurement points, or, a low pass filter may be applied in order to smooth the results of the reconstruction. Reconstructed waveforms may present time delays relative to the capacitive sensor signal used as reference for comparison purposes. Thus, for the sensor comparison presented in Section 4, a lag detection and compensation algorithm was also implemented.

\section{Experimental results}

In order to test the attainable resolution of the DOFI method in laboratory conditions, a prototype was built (Fig. 4). The prototype setup is composed of two HITACHI Fabry-Perot (FP) HL7851G laser diodes with an adjustable 
focusing lens. Previous to the test the lasers were conditioned according to Section 2. The measurement laser with $\lambda_{m}=778.78 \pm 0.10 \mathrm{~nm}$ was mechanically attached to a PI-LISA piezoelectric displacement stage with nominal amplitude of $38 \mu \mathrm{m}$. The reference laser with $\lambda_{r}=784.95 \pm 0.10 \mathrm{~nm}$ was placed facing the reference stage at an approximate distance of 10 $\mathrm{cm}$. A second PI-LISA stage with an internal capacitive sensor and nominal displacement amplitude of $25 \mu \mathrm{m}$ was chosen as target. The capacitive sensor within the stages has a resolution of $2 \mathrm{~nm}$ and is used for validation purposes. The stage was placed approximately at $10 \mathrm{~cm}$ from the measuring laser. All the electrical signals are pre-conditioned using the electronics described in Section 3 and are acquired using a Tektronics DP02024 oscilloscope with $125 \mathrm{kS}$ window with a length of $0.4 \mathrm{~s}$. Once acquired the signal is pre-filtered with a low pass filter with cut-off frequency of $55 \mathrm{kHz}$.

The displacement of the target was chosen as a sinusoidal motion with fixed peak to peak amplitude of $40 \mathrm{~nm}$ and frequency range

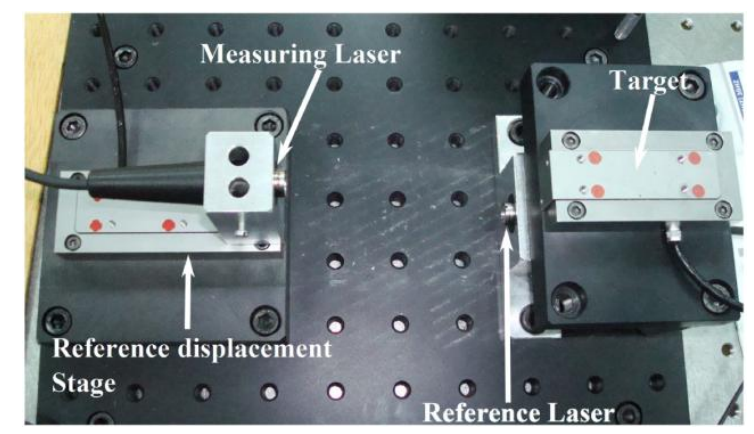

Fig. 4. Schematic representation of electronic card requirements for DOFI acquisition with wavelength stabilization.

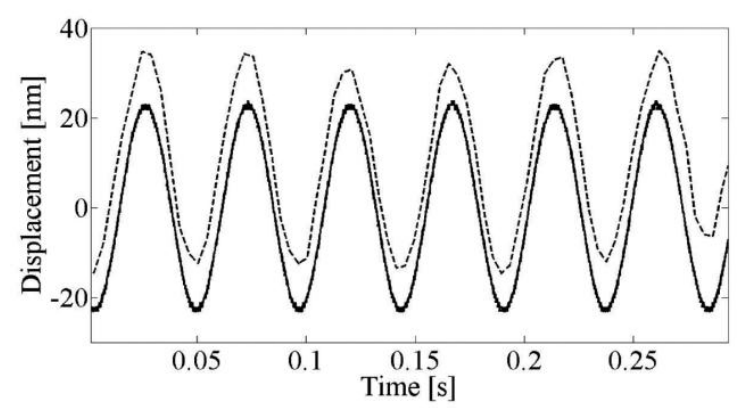

Fig. 5. Reconstruction of a 40nm-amplitude sinusoidal displacement. Dotted lines DOFI measured values, solid line reference values running from 5 to $50 \mathrm{~Hz}$. The measurements were performed 10 times for each frequency to test the repeatability of the measurement.

When compared with capacitive sensor readings, as shown in Fig. 5, the reconstructed waveforms show average errors in the order of $1 \mathrm{~nm}$ and standard deviations in the order of 5 $\mathrm{nm}$. A summary of the average errors and average standard deviation values at different frequencies is shown in Table I.

The set of results shows that experimentally it is possible to reconstruct displacements with a resolution of $\lambda / 100$. Similar results were also observed when modulation depths of up to $90 \%$ of the signal were used.

As observed, a decrease of 10 may be observed in the present setup when the experimental accuracy is compared to the theoretical resolution described in [15]. This difference is attributed to noise effects in the signal due to the amplification circuit and to mechanical couplings present in the setup.

\section{Conclusion}

In this paper we have presented experimental results for the DOFI technique. Experimental results have shown errors in the order of $1 \mathrm{~nm}$, and standard deviation values in the order of 5 $\mathrm{nm}$, showing a $\lambda / 100$ resolution. Nevertheless, a decrease in the attainable resolution in a factor of 10 has been found with respect to the theoretical results presented in [15], where it is shown that for sampling conditions similar as the ones depicted in Section 2, and, considering other effects on the SMI signal such as a decrease on the $C$ value and the increase of white noise, it

Table I

Average error and standard deviation between OFI interpolated values and capacitive sensor response for sinusoidal displacements at different frequencies $F$. The uncertainty of the measurement is measured using a $1 \sigma$ standard deviation from the measurements. All the values are in $\mathrm{nm}$.

\begin{tabular}{|c|c|c|c|c|}
\hline $\boldsymbol{F}[\mathrm{Hz}]$ & Avg. Err. & Unc. & Std. Dev. & Unc. \\
\hline 5 & 1.05 & \pm 0.70 & 6.91 & \pm 1.41 \\
\hline 10 & 0.78 & \pm 0.69 & 4.14 & \pm 2.58 \\
\hline 20 & 0.45 & \pm 0.15 & 2.71 & \pm 0.51 \\
\hline 50 & 0.46 & \pm 0.47 & 4.88 & \pm 2.11 \\
\hline
\end{tabular}


should be possible to perform measurements with attainable resolution in the subnanometre scale (e.g. $\lambda / 1000$ ). While there might be several reasons to explain the decrease of resolution, we are inclined to think that the deviation is produced in some point of the applied fringe detection algorithm. Further work in this subject is needed in order to reduce the uncertainty of this process.

We have also presented a short summary of the electronic requirements to build a DOFI signal acquisition system. The current mirror used in the conditioning card is specially important for the correct reconstruction of the DOFI signal, since enables to provide long term stability to the wavelength of the lasers. VCSEL devices could possibly be used without this kind of feedback because of its better wavelength stability. Nevertheless, a wavelength control such as the one described can also help to improve the accuracy of the measurement.
Besides the development of targeted applications, further work will be focused on the design of a complete acquisition and processing system, as well as the improvement of current measurement algorithms to produce on-line measurements.

\section{Acknowledgements}

The authors would like to thank the Spanish Ministry of Science and Innovation for the funding provided by the Plan Nacional de I+D+i through project DPI2011-25525. Francisco J. Azcona would also like to thank the Department d'Universitats, Recerca i Societat de la Informació of Generalitat de Catalunya for its funding through the pre-doctoral grant 2012FIB1-00240. 